\title{
Patient-Defined Visit Priorities in Primary Care: Psychosocial Versus Medically-Related Concerns
}

\author{
Eilann Clare Santo, MD, Michelle TuongVi Vo, Connie S. Uratsu, RN, MS, CNS, and \\ Richard William Grant, MD, MPH
}

Background: Primary care providers (PCPs) are often challenged to address multiple patient concerns during time-limited visits. The need for PCPs to limit the number of issues addressed may have a negative impact on discussion of patient-defined visit priorities.

Methods: Using data from a recent clinical trial (Aligning Patients and Providers, ClinicalTrials.gov: NCT02707146), we examined the association between patient-defined visit priorities and subsequent provider actions taken during and after the visit. We tested the hypothesis that psychosocial concerns (eg, stress, anxiety, caregiving demands) are less likely to be addressed than traditional medical concerns.

Results: We analyzed 147 patient-defined visit priorities submitted just before the visit by 109 patients (mean age, $\mathbf{5 9 . 0} \pm \mathbf{1 2 . 7}$ years; including $\mathbf{7 3 . 4 \%}$ women, $\mathbf{4 7 . 7 \%}$ non-White race/ethnicity). Nearly one quarter of patient-defined visit priorities were related to psychosocial concerns $(35 / 147 ; 23.8 \%)$. In models adjusting for age, gender, race/ethnicity, and familiarity with PCP, patients' psychosocial priorities were significantly less likely than medical priorities to be addressed during the visit (63\% vs. 88\%; adjusted odds ratio [aOR], $0.16 ; 95 \% \mathrm{CI}, 0.06$ to $0.41 ; P<.001)$, to receive clinical action $(51 \%$ vs. $82 \%$; a OR, $0.15 ; 95 \%$ CI, 0.06 to $0.38 ; P<.001$ ), or to receive post visit information from the primary care doctor $(17 \%$ vs. $32 \%$; a OR, $0.39 ; 95 \% \mathrm{CI}, 0.14$ to $1.08 ; P=.07)$.

Conclusions: Patient-defined psychosocial priorities are less likely to be addressed during (or immediately after) primary care visits compared with patient-defined medical priorities. (J Am Board Fam Med 2019;32:513-520.)

Keywords: Communication, Mental Health, Patient-Centered Care, Physician-Patient Relations, Primary Health Care, Primary Care Physicians, Social Determinants of Health

Patients frequently experience psychosocial concerns such as financial or housing insecurity, caregiving or work-related stress, and mental health symptoms including anxiety or subclinical depression that can significantly interfere with their quality of life. ${ }^{1,2}$ Prior observational studies have suggested that psychosocial issues are less fre-

This article was externally peer reviewed.

Submitted 19 December 2018; revised 26 March 2019; accepted 31 March 2019.

From the Department of Internal Medicine(ECS), Division of Research (MTV, CSU, RWG), Kaiser Permanente Northern California, Oakland, CA.

Funding: Research reported in this publication was funded through a Patient-Centered Outcomes Research Institute (PCORI) Award (CDR-1403-11992) and National Institute of Diabetes and Kidney Diseases (NIDDK K24DK109114).

Conflict of interest: none declared.

Corresponding author: R. W. Grant, MD, MPH, Division of Research, Kaiser Permanente Northern California, 2000 Broadway, Oakland, CA 94612 (E-mail: Richard.W.Grant@ kp.org). quently addressed during primary care visits due to time constraints, the more complex nature of these issues, and lack of available resources. ${ }^{3-6}$ This failure to address the full spectrum of patient needs is problematic given that factors such as financial concerns, stress, and anxiety have been shown to negatively impact health outcomes, leading to lower functional status, earlier mortality, increased rates of medical complications, and higher incidence of acute hospitalization. ${ }^{7-12}$

Primary care providers (PCPs) must balance management of acute and chronic medical conditions while also addressing an increasing number of quality and guideline-based metrics. ${ }^{13-15}$ Multiple competing demands and limited time during primary care visits represent significant barriers to successful patient-provider communication and collaboration. ${ }^{16-18}$ With the aging of the general population, patients present for care with a rising 
number of concurrent chronic medical conditions. ${ }^{19,20}$ As a result, visit agendas may be largely defined by PCPs, and patients may not always have the opportunity to voice their concerns and may leave with unmet needs. ${ }^{21,22}$

Because primary care visits typically involve discussion of multiple different concerns (eg, patient symptoms, test results, behavior changes, recommended screenings), understanding the relationship between patient-defined priorities and subsequent provider actions can provide insight into how agendas are set during visits. We had the unique opportunity to examine patient-defined visit priorities among a group of primary care patients participating in a multi-site, randomized clinical trial. In this Aligning Patients and Providers trial, patients in the intervention arm were guided to identify their top 1 or 2 visit priorities in the waiting room just before their primary care visit using a "Visit Planner" tool. ${ }^{23}$ Patients received a article print-out listing their top visit priorities then proceeded to their visit. For this current report, we examine the relationship between types of patient-defined visit priorities and corresponding clinical actions arising from the visit. We tested the hypothesis that providers would be less likely to address patient-identified psychosocial concerns compared with medically related patient priorities.

\section{Methods}

\section{Setting}

Our analysis was conducted using data from the Aligning Patients and Providers study (APP; ClinicalTrials.gov: NCT02707146), a randomized controlled trial conducted within Kaiser Permanente Northern California (KPNC). KPNC is a nonprofit, integrated health care delivery system that provides care for over 4.1 million members throughout Northern California.

Full details of the trial have been published elsewhere. ${ }^{24}$ Briefly, primary care physicians were recruited for the clinical trial between June 2015 and February 2016 from 7 primary care practices. $\mathrm{Pa}$ tient clinical trial eligibility was based on having a current gap in evidence-based primary care, defined as being due for preventive cancer screening (breast, cervical, and colorectal); having suboptimal medication adherence (for treatment of diabetes, hypertension, or dyslipidemia); being a current smoker; or having suboptimal chronic disease control (defined as elevated Hemoglobin A1c [HbA1c], systolic blood pressure, or Low-Density Lipoprotein [LDL]-cholesterol; or being overdue for osteoporosis treatment). This broad array of potential inclusion criteria resulted in a fairly representative cohort of general primary care patients. Patients with a diagnosis of dementia, psychosis, or other medical conditions which could impair participation were excluded from the study. Patients were initially contacted by phone for participation in the study after they had scheduled an appointment. During this phone call, the research assistant arranged to meet the patient in the waiting room before the visit, where written informed consent was obtained. The study was approved by the institutional review board of the Kaiser Foundation Research Institute.

\section{Patient-Identified Visit Priorities}

Our current analysis focuses on data collected from patients enrolled in the intervention arm of the APP trial. Using a tablet-based tool in the waiting room ("Visit Planner"), these patients viewed a 30 -second video clip on the importance of mentioning their priorities at the beginning of the visit and then selected their top 1 or 2 visit priorities from a list of 6 prespecified categories. Within each category, patients could further select from up to 5 subcategories and/or add free text to further clarify the nature of the priority (Table 1). After making their choices, patients received a 1-page article print-out with their selections from research assistant before going in to the examination room. The patient-priorities were not entered by staff into the medical record.

For our analysis, responses were grouped into 2 mutually exclusive categories of psychosocial versus medical priorities based on patient category selection and any accompanying free text responses. Priorities were considered psychosocial if patients identified: 1) 'I am feeling anxious or depressed,' 2) 'Drug or alcohol concerns,' 3) 'Caregiving issues,' 4) 'Family concerns,' or 5) 'Stress at home or work.' Free text responses with specific mention of depression, anxiety, stress, family concerns, or substance use were also categorized as psychosocial priorities. Priorities where patients indicated specific medical concerns (eg, 'back pain,' 'cough,' 'diabetes') were categorized as medical priorities. Priorities that could not be categorized as either psychosocial or medically-related (eg, 'New problem' or 'Old problem' without any explanatory text) were excluded from our analysis. 
Table 1. Visit Priority Options (6 Main Categories and Associated Subcategories) and their Corresponding Psychosocial Versus Medical Designations for This Study

\begin{tabular}{|c|c|c|}
\hline 6 Main Categories & Associated Subcategories & Designation \\
\hline \multirow[t]{3}{*}{ Stress at home or work } & Caregiving issues & Psychosocial \\
\hline & Family concerns & Psychosocial \\
\hline & Financial concerns & Psychosocial \\
\hline \multirow[t]{3}{*}{ A personal concern } & Anxious or depressed & Psychosocial \\
\hline & Drug or alcohol concerns & Psychosocial \\
\hline & My personal safety* & \\
\hline \multirow[t]{3}{*}{ Medicines } & Medicines cost too much & Psychosocial \\
\hline & Problems with side effects & Medical \\
\hline & Stopped taking & Medical \\
\hline \multirow[t]{4}{*}{ Need something from my doctor } & Referral to a specialist & Medical \\
\hline & Medication refill & Medical \\
\hline & Blood test, $\mathrm{x}$ ray, or other test & Medical \\
\hline & Form filled out & Medical \\
\hline \multirow[t]{3}{*}{ New problem } & I'm feeling anxious or depressed & Psychosocial \\
\hline & I've noticed something new* & \\
\hline & I'm having trouble with my usual activities* & \\
\hline \multirow[t]{3}{*}{ Old problem } & Something has changed* & \\
\hline & Need more explanation* & \\
\hline & I'm not getting better from last time* & \\
\hline
\end{tabular}

*Included in analysis if free text clearly defined the patient's priority as medical or psychosocial concern.

\section{Study Outcomes}

We used structured electronic chart review of visit progress notes, after-visit summaries, orders placed during the visit, specialty referrals, and postvisit email messages between patient and provider to determine whether the provider addressed the patient's visit priorities.

The main outcome of interest was concordance between patient-defined priority and provider response. Concordance was examined in 3 domains: 1) provider documentation of the patient-defined priority in the progress note or patient problem list, 2) evidence of action or follow up through relevant referral, documentation of in-office counseling, ordering of relevant lab tests, imaging studies, or therapeutics, and 3) provision of information about the patient-defined priority through a primary care after-visit summary or a secure message to the patient with specific reference to the patient priority within 72 hours of the visit.

\section{Statistical Analyses}

We examined patient-provider concordance comparing psychosocial versus medically related patient priorities using $\chi^{2}$ tests with a significance level of 0.05 and 2 -sided hypothesis testing. In secondary analyses, we also examined patient-provider concordance stratified by history of patient mental health disorder and by primary care physician gender. Mental health disorders were defined by International Classification of Diseases (ICD) 10 diagnosis (anxiety disorder, major depressive disorder, post-traumatic stress disorder, Attention-Deficit/ Hyperactivity Disorder, bipolar disorder type 1 or 2 , or addiction to alcohol or illicit substances).

We constructed logistic regression models (SAS GENMOD) to examine odds of concordance by priority type (psychosocial vs medically-related) after adjusting for potential confounding variables including patient and provider gender, patient age and race, and patient-PCP familiarity defined as new to PCP $(<18$ months) versus established relationship ( $\geq 18$ months). We applied generalized estimating equations to adjust for the nonindependence of multiple priorities for some patients. All analyses were performed using (SAS version 9.3, SAS Institute, Cary NC).

\section{Results}

\section{Cohort Characteristics}

Our cohort included 109 patients with self-identified psychosocial or medical visit priorities. Mean 
Table 2. Characteristic of Patients with and without any Psychosocial Visit Priorities

\begin{tabular}{|c|c|c|c|c|}
\hline & $\begin{array}{l}\text { All Patients } \\
(\mathrm{n}=109)\end{array}$ & $\begin{array}{l}\text { Any Psychosocial } \\
\quad(\mathrm{n}=31)\end{array}$ & $\begin{array}{l}\text { Only Medical } \\
\quad(\mathrm{n}=78)\end{array}$ & $P$-Value \\
\hline Age, years: Mean (SD) & $59.0(12.7)$ & $56.8(13.3)$ & $59.9(12.4)$ & .3 \\
\hline Range & 30 to 80 & 34 to 79 & 30 to 80 & \\
\hline \multicolumn{5}{|l|}{ Age, years, \% } \\
\hline 30 to 59 & $46(42.2)$ & $15(48.4)$ & $31(39.7)$ & .4 \\
\hline 60 to 80 & $63(57.8)$ & $16(51.6)$ & $47(60.3)$ & \\
\hline \multicolumn{5}{|l|}{ Gender, \% } \\
\hline Women & $80(73.4)$ & $24(77.4)$ & $56(71.8)$ & .5 \\
\hline Men & $29(26.6)$ & $7(22.6)$ & $22(28.2)$ & \\
\hline \multicolumn{5}{|l|}{ Race/ethnicity, \% } \\
\hline Non-white & $52(47.7)$ & $16(51.6)$ & $36(46.2)$ & 6 \\
\hline White & $57(52.3)$ & $15(48.4)$ & $42(53.8)$ & \\
\hline \multicolumn{5}{|l|}{ New to PCP, \% } \\
\hline Yes & $46(42.2)$ & $15(48.4)$ & $31(39.7)$ & .4 \\
\hline No & $63(57.8)$ & $16(51.6)$ & $47(60.3)$ & \\
\hline Current medications, mean (SD) & $2.5(2.5)$ & $2.5(2.6)$ & $2.5(2.5)$ & .9 \\
\hline Medical conditions, mean (SD) & $1.4(0.6)$ & $1.3(0.5)$ & $1.4(0.6)$ & .5 \\
\hline \multicolumn{5}{|l|}{ Care from, $\%$} \\
\hline Female PCP & $81(74.3)$ & $25(80.6)$ & $56(71.8)$ & .3 \\
\hline Male PCP & $28(25.7)$ & $6(19.4)$ & $22(28.2)$ & \\
\hline
\end{tabular}

PCP, primary care provider; SD, standard deviation.

age was $59.0( \pm 12.7)$ years and $73.4 \%$ were women. A slight majority of patients identified as non-Hispanic white $(52.3 \%)$. Patients were taking $2.5( \pm 2.5)$ medications. Most patients received care from a female primary care physician $(74.3 \%)$ and most patients had the same gender as their physician $(71.5 \%$ gender concordance). We found no statistically significant baseline differences between patients with any psychosocial concerns compared with patients with only medical concerns (Table 2).

Patients listed a total of 147 unique priorities which were included in our analysis. Most patients identified a single visit priority $(77 / 109 ; 71 \%)$. Nearly one quarter of all priorities $(35 / 147 ; 23.8 \%)$ were categorized as psychosocial concerns. Of these, the most common were anxiety and depression (54\%) and general psychosocial stress (43\%). The most frequently patient-identified medical priorities were related to musculoskeletal $(26 \%)$ or cardiovascular (12\%) concerns.

\section{Patient-Provider Concordance by type of Patient Visit Priority}

Psychosocial priorities were less likely to be addressed by the primary care provider compared with medically related priorities. More specifically, psychosocial priorities were less often documented in the chart $(62.9 \%$ vs. $88.4 \% ; P<.005)$, less often subject to clinical action or follow-up $(51.4 \%$ vs. $82.1 \% ; P<.005)$, and less often included in post visit information from the primary care doctor (17.1\% vs. $32.1 \% ; P=.09)$ compared with medically related health priorities (Figure 1). Results remained similar when stratified by history of mental health disorder, provider gender, or whether patient and provider were of the same gender.

These concordance differences between psychosocial and medically related priorities remained after adjusting for patient and provider gender, age, patient race, history of mental health disorder, and familiarity between patient and provider. Psychosocial priorities were less likely to be addressed by the primary care provider $(62 \%$ vs. $88 \%$; adjusted odds ratio [aOR], 0.16 ; $95 \% \mathrm{CI}, 0.06$ to $0.41 ; P<$ $.001)$, less likely to receive clinical action $(54 \%$ vs. $82 \%$; aOR, $0.15 ; 95 \%$ CI, 0.06 to $0.38 ; P<.001$ ), and less likely to receive post visit information from the primary care doctor $(17 \%$ vs. $32 \%$; aOR, 0.39 ; $95 \% \mathrm{CI}, 0.14$ to $1.08 ; P=.07)$ compared with medically related priorities. Of the other variables considered in our models, only higher patient age 
Figure 1. Bars represent proportion of psychosocial (dark) or medically-related (light) patient-defined visit priorities that were addressed by provider, either through documentation of the patient-defined visit priority in the chart $(62.9 \%$ vs $88.4 \%, p<.005)$, taking clinical action $(51.4 \%$ vs. $82.1 \%, p<.005)$, or following up with the patient after the visit with related information $(17.1 \%$ vs $32.1 \%, p=0.09)$.

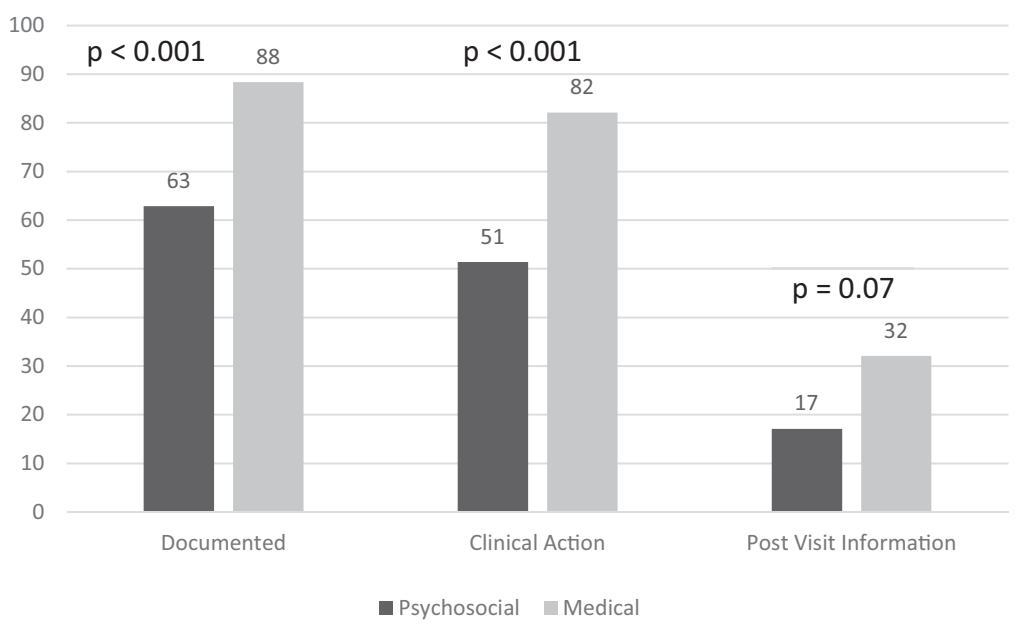

was independently associated with lower odds of a patient-identified priority being addressed by the primary care provider $(76 \%$ vs. $91 \%$ for age 60 to 80 vs. 30 to 59 years; aOR, $0.31 ; 95 \% \mathrm{CI}, 0.11$ to $0.82 ; P<.02)$.

\section{Conclusions}

Psychosocial concerns are a frequent visit priority for patients in primary care. Prior research has suggested that these concerns may receive less attention than medically defined problems during primary care visits. ${ }^{25,26}$ In prior studies, identification of psychosocial concerns was by physicians rather than by patients. We had the unique opportunity to examine primary care interactions based on patient-defined visit priorities. These priorities were elicited in the waiting room as part of the APP clinical trial, an intervention that included a brief (30-second) video clip encouraging patients to bring up their visit priorities at the beginning of the visit and an easy process for patients to select their priorities, which were then printed out for them before they entered the examination room.

Through review of these patient-identified priorities during primary care visits and the outcomes of these visits, we found that patient-defined psychosocial priorities were markedly less likely to be addressed by primary care physicians than medically related priorities. Our results extend prior research that providers are more likely to address medical concerns as opposed to psychosocial prior- ities $^{3-5,27-29}$ by demonstrating that this disparity exists even when patients themselves have identified these concerns as top visit priorities.

There are likely several different contributors to this disparity between how psychosocial and medically related priorities are addressed in primary care. Providers receive little training in how to talk to patients about concerns such as food or housing insecurity, financial concerns, or caregiver burnout or fatigue. For these reasons, some physicians may perceive medical concerns as more appropriate targets for their care. Providers may also be less likely to initiate discussion regarding more complex psychosocial issues, especially among patients with chronic conditions, as they tend to have less concrete solutions. ${ }^{30}$ Providers may also lack the necessary resources (eg, available mental health consultants or social workers who can help with financial issues) to direct toward patients with nonmedical needs. These psychosocial needs are also generally less amenable to actions such as ordering tests, giving lifestyle advice, and prescribing medicines with which providers are generally most comfortable.

One strength of our analytic approach was to examine multiple domains of provider action: formal documentation, different tangible clinical actions, and follow-up contact with the patient. It is perhaps not surprising that patient-prioritized psychosocial issues were less likely to be documented during the visit, as providers may limit documen- 
tation to details that support billing requirements. To the extent that the psychosocial priorities raised by patients may be helpful for other clinicians reading the chart in future encounters, our results suggest that this may be a missed opportunity. Indeed, evidence has shown that patient-centered carewhich would include addressing patient-defined psychosocial needs-can improve care and reduce costs. $^{31}$

A secondary finding from our analysis was the independent negative association of patient age with patient-provider concordance on visit priorities. This can likely be explained by the fact that older patients have a larger number of chronic medical conditions ${ }^{32}$ and providers may be more strained for time to address patient priorities. ${ }^{33}$ Given the ageing of the primary care population, this finding may have important implications for growing the need to improve awareness of psychosocial issues in older patients. Current literature suggests that to circumvent this issue, elderly patients may benefit from family member or companion presence during primary care visits to help more effectively advocate for the patient's concerns. $^{34}$

Prior research has also shown that female physicians tend to be more patient-centered than male physicians and are more likely to address patient concerns, particularly when discussing psychosocial matters. ${ }^{35}$ In contrast to these prior studies, we found no correlation between gender of primary care provider and concordance with patient-defined priorities. This association, however, has only been previously described in settings where providers had to directly elicit patient priorities, rather than receiving them from the patient, and may speak more to communication style discrepancies between genders rather than patients being forthcoming with their priorities. ${ }^{36}$

Several limitations of our study deserve mention. Our concordance assessment was based on documented priorities and actions and thus does not capture conversations that may have been conducted during the visit. Indeed, although patients were coached to communicate their visit priorities at the beginning of the visit, we cannot be sure that patients voiced these priorities. In addition, current electronic health record (EHR) systems are typically better designed to facilitate coding of medical rather than psychosocial concerns. Nonetheless, the disparities in documentation, clinical actions, and postvisit messaging captured through structured chart review all support our conclusion that psychosocial issues are less likely to be meaningfully addressed in primary care than medically related ones. In addition, we were unable to capture whether more pressing clinical matters such as an acute medical problem superseded patient priorities, thus making concordance less likely. Our tabulation of priorities from our study participants may not be generalizable to other populations, both because all KPNC patients are insured and because participation in the APP clinical trial may have facilitated recognition of psychosocial priorities. Finally, due to the relatively small sample sizes, we were unable to examine concordance by specific concerns within our 2 mutually exclusive categories.

Our results highlight the difficulties that patients face in having psychosocial needs addressed during primary care visits. Based on prior studies, we speculate that the multitude of competing clinical demands and limited resources for psychosocial care may be contributors to the results we found. Although we could not address this question directly in our analysis, our results suggest potential solutions to this problem may include improved provider training and increased care coordination with other primary care team members. Indeed, many primary care practices have begun to include a behavioral medicine specialist on the care team to help support patients with psychosocial issues. Future efforts to improve care should focus not only on helping physicians discuss patient priorities at the outset of the visit to help maximize visit efficiency and improve patient experience, ${ }^{37,38}$ but also to help bolster clinic- and community-based resources to provide psychosocial support to $\mathrm{pa}$ tients. ${ }^{39,40}$

To see this article online, please go to: http://jabfm.org/content/ 32/4/513.full.

\section{References}

1. Uphoff EP, Pickett KE, Cabieses B, et al. A systematic review of the relationships between social capital and socioeconomic inequalities in health: A contribution to understanding the psychosocial pathway of health inequalities. Int J Equity Health 2013;12:54.

2. Walker ER, Druss BG. Cumulative burden of comorbid mental disorders, substance use disorders, chronic medical conditions, and poverty on health among adults in the U.S.A. Psychol Health Med 2017;22:727-35. 
3. Glied S. Too little time? The recognition and treatment of mental health problems in primary care. Health Serv Res 1998;33:891-910.

4. Corrigan PW, Mittal D, Reaves CM, et al. Mental health stigma and primary health care decisions. Psychiatry Res 2014;218:35-8.

5. Tai-Seale M, McGuire T, Colenda C, et al. Twominute mental health care for elderly patients: Inside primary care visits. J Am Geriatr Soc 2007;55:1903-11.

6. Peltenburg M, Fischer JE, Bahrs O, et al. The unexpected in primary care: A multicenter study on the emergence of unvoiced patient agenda. Ann Fam Med 2004;2:534-40.

7. Valtorta NK, Kanaan M, Gilbody S, et al. Loneliness and social isolation as risk factors for coronary heart disease and stroke: Systematic review and meta-analysis of longitudinal observational studies. Heart 2016;102:1009-16.

8. Hanratty B, Stow D, Collingridge Moore D, et al. Loneliness as a risk factor for care home admission in the English Longitudinal Study of Ageing. Age Ageing 2018;47:896-900.

9. Phelan JC, Link BG, Diez-Roux A, et al. "Fundamental causes" of social inequalities in mortality: A test of the theory. J Health Soc Behav 2004;45:265-85.

10. Link BG, Phelan J. Social conditions as fundamental causes of disease. J Health Soc Behav 1995;35:80-94.

11. Patel MR, Piette JD, Resnicow K, et al. Social determinants of health, cost-related nonadherence, and cost-reducing behaviors among adults with diabetes: Findings From the National Health Interview Survey. Med Care 2016;54:796-803.

12. Walker ER, Pratt LA, Schoenborn CA, et al. Excess mortality among people who report lifetime use of illegal drugs in the United States: A 20-year follow-up of a nationally representative survey. Drug Alcohol Depend 2017;171:31-8.

13. Tinetti ME, Fried TR, Boyd CM. Designing health care for the most common chronic condition-Multimorbidity. JAMA 2012;307:2493-4.

14. Vogeli C, Shields AE, Lee TA, et al. Multiple chronic conditions: Prevalence, health consequences, and implications for quality, care management, and costs. J Gen Intern Med 2007;22:391-5.

15. Pollak KI, Krause KM, Yarnall KS, et al. Estimated time spent on preventive services by primary care physicians. BMC Health Serv Res 2008;8:245.

16. Ostbye T, Yarnall KS, Krause KM, et al. Is there time for management of patients with chronic diseases in primary care? Ann Fam Med 2005;3:209-14.

17. Abbo ED, Zhang Q, Zelder M, et al. The increasing number of clinical items addressed during the time of adult primary care visits. J Gen Intern Med 2008; 23:2058-65

18. Parchman ML, Romero RL, Pugh JA. Encounters by patients with type 2 diabetes-Complex and demanding: An observational study. Ann Fam Med 2006;4:40-5.
19. Lochner KA, Cox CS. Prevalence of multiple chronic conditions among Medicare beneficiaries, United States, 2010. Prev Chronic Dis 2013;10:E61.

20. Schneider KM, O’Donnell BE, Dean D. Prevalence of multiple chronic conditions in the United States' Medicare population. Health Qual Life Outcomes 2009;7:82.

21. Kowalski CP, McQuillan DB, Chawla N, et al. 'The Hand on the Doorknob': Visit agenda setting by complex patients and their primary care physicians. J Am Board Fam Med 2018;31:29-37.

22. Barry CA, Bradley CP, Britten N, et al. Patients' unvoiced agendas in general practice consultations: Qualitative study. BMJ 2000;320:1246-50.

23. Lyles CR, Altschuler A, Chawla N, et al. Usercentered design of a tablet waiting room tool for complex patients to prioritize discussion topics for primary care visits. JMIR Mhealth Uhealth 2016;4: e108.

24. Grant RW. Lyles C, Uratsu CS, et al. Visit planning using a waiting room health IT tool: The aligning patients and providers randomized clinical trial. Ann Fam Med 2019;17:141-9.

25. Rocque R, Leanza Y. A systematic review of patients' experiences in communicating with primary care physicians: Intercultural encounters and a balance between vulnerability and integrity. PLoS One 2015; 10:e0139577.

26. Lim CY, Berry ABL, Hirsch T, et al. Understanding what is most important to individuals with multiple chronic conditions: A qualitative study of patients' perspectives. J Gen Intern Med 2017;32:1278-84.

27. Tai-Seale M, Bramson R, Bao X. Decision or no decision: How do patient-physician interactions end and what matters? J Gen Intern Med 2007;22:297302 .

28. Shires DA, Stange KC, Divine G, et al. Prioritization of evidence-based preventive health services during periodic health examinations. Am J Prev Med 2012;42:164-73.

29. Tai-Seale M, McGuire TG, Zhang W. Time allocation in primary care office visits. Health Serv Res 2007;42:1871-94.

30. Bohlen K, Scoville E, Shippee ND, et al. Overwhelmed patients: A videographic analysis of how patients with type 2 diabetes and clinicians articulate and address treatment burden during clinical encounters. Diabetes Care 2012;35:47-9.

31. Bertakis KD, Azari R. Patient-centered care is associated with decreased health care utilization. J Am Board Fam Med 2011;24:229-39.

32. Walke LM, Gallo WT, Tinetti ME, et al. The burden of symptoms among community-dwelling older persons with advanced chronic disease. Arch Intern Med 2004;164:2321-4.

33. Fried TR, Tinetti ME, Iannone L. Primary care clinicians' experiences with treatment decision mak- 
ing for older persons with multiple conditions. Arch Intern Med 2011;171:75-80.

34. Laidsaar-Powell RC, Butow PN, Bu S, et al. Physician-patient-companion communication and decision-making: A systematic review of triadic medical consultations. Patient Educ Couns 2013;91:3-13.

35. Roter DL, Hall JA. Physician gender and patientcentered communication: A critical review of empirical research. Annu Rev Public Health 2004;25:497519.

36. Roter DL, Hall JA, Aoki Y. Physician gender effects in medical communication: A meta-analytic review. JAMA 2002;288:756-64.

37. Grant RW, Adams AS, Bayliss EA, et al. Establishing visit priorities for complex patients: A summary of the literature and conceptual model to guide inno- vative interventions. Healthc (Amst) 2013;1(3-4): 117-22.

38. Hemming P, Revels JA, Tran AN, et al. Identifying core curricular components for behavioral health training in internal medicine residency: Qualitative interviews with residents, faculty, and behavioral health clinicians. Int J Psychiatry Med 2019;54:188202.

39. Blickem C, Kennedy A, Vassilev I, et al. Linking people with long-term health conditions to healthy community activities: Development of Patient-Led Assessment for Network Support (PLANS). Health Expect 2013;16:e48-e59.

40. Rich EC, Lipson D, Libersky J, et al. Organizing care for complex patients in the patient-centered medical home. Ann Fam Med 2012;10:60-2. 